\title{
A nonlinear physics model based on extended synergetics for the flow of infant actions during infant-mother face-to-face communication
}

\author{
T.D. Frank \\ Department of Psychology, CESPA, University of Connecticut, Storrs, Connecticut, United States \\ E-mail: till.frank@uconn.edu
}

Copyright (C)2014 T.D. Frank. This is an open access article distributed under the Creative Commons Attribution License, which permits unrestricted use, distribution, and reproduction in any medium, provided the original work is properly cited.

\begin{abstract}
The temporal ordering or flow of infant actions in the context of infant-mother face-to-face communication is discussed from a nonlinear physics perspective. The actions are considered as spatio-temporal patterns that emerge due to self-organization. The presence of a particular action is described in terms of a finite amplitude of the respective pattern. In line with benchmark models from synergetics and population dynamics, nonlinear amplitude equations that determine the evolution of the infant actions are defined. The model is extended to take selfinhibition and forgetting into account. More precisely, it is shown that the flow of infant actions during infant-mother communication can be explained by assuming that the infant behavior inhibits itself such that after a certain period of time the performed behavior becomes unstable and a transition towards a new behavior occurs. The proposed model is applied to literature data about three minute periods of infant-mother face-to-face communication. Since the model parameters determine the characteristic properties of the flow of infant actions, the conclusion is drawn that changes of the flow characteristics observed on the behavioral level during infant development correspond to changes of parameters on the neurobiological level that happen on the relative slow time scale of infant maturation.
\end{abstract}

Keywords: Extended Synergetics, Flow of Actions, Infant-Mother Communication, Lotka-Volterra Model, Nonlinear Physics

\section{Introduction}

Nonlinear physics has been a useful tool for describing phenomena in the life sciences in general and in psychology in particular $[1,2]$. In this context, nonlinear physics models have been proposed that account for the multistability of infant motor actions [3,4] and child leisure activities [5]. Accordingly, in order to explain that the repertoire of motor actions that can be performed by an infant at a given age changes during infant development, it is assumed that motor actions are self-organized spatio-temporal patterns with amplitudes whose growth factors depend on developmental control parameters. Likewise, in order to arrive at a nonlinear physics model that can produce a sequence of leisure-related actions (i.e., a flow of actions) it has been suggested to regard these actions as selforganized patterns. In order to address temporal ordering aspects, it has been proposed that the pattern amplitudes are subjected to self-inhibition. Explicitly, the self-inhibition may affect the aforementioned growth parameters of the amplitudes.

While the synergetic approach allows us to understand the emergence of perceptual-cognitive-behavioral patterns 
from a self-organization perspective, it has been shown that the concept of self-inhibitory perceptual-cognitivebehavioral patterns requires to supplement the synergetic approach with a parameter dynamics. In other words, while the synergetic model addresses pattern formation in self-organized systems when control parameters are changed externally (e.g., by the experimenter), the synergetic model as such does not take the self-regulation of system parameters into consideration. Let us be clear about the key conceptual issue under consideration: the self-regulation of system parameters. Let us provide a formal definition in this regard.

Definition 1.1 Self-regulation of a system parameter in the context of self-organizing system

When a system parameter of a self-organizing system changes

due to the fact that the system is in a particular self-organized state

then we say that the system exhibits a self-regulation of the system parameter.

If the definition given in Eq. (1) applies to a system then the system may be described by means of extended synergetics. Extended synergetic models have been studied to describe selective attention and scene decomposition $[6,7]$, the oscillatory perception of ambivalent figures $[8,9]$ and the counterpart on the acoustic level, the so-called verbal transformation effect $[10,11]$. Moreover, the extended synergetic approach has been used to address transitions between perceptual-cognitive-behavioral patterns in the case of transitions that exhibit negative hysteresis $[12,13]$. Theoretical considerations suggest that phenomena such as priming, on the one hand, and retrievalinduced forgetting, on the other, have an underlying system parameter dynamics component and consequently may be studied in the context of extended synergetics [14, 15]. Medication non-adherence and relapse in bipolar disorder patients may be explained by postulating a habituation process that in turn is modeled by a self-regulated parameter dynamics [16].

Finally, as mentioned above, a nonlinear physics model for the temporal ordering of leisure activities of children was proposed in an earlier study [5]. According to that model, transitions between leisure activities happen due to an inhibitory effect of the performed activity on itself. The self-inhibition effect is modeled in terms of a parameter dynamics again. While the model was introduced in the aforementioned earlier study [5], it was not worked out in detail. Therefore, after introducing the general model based in extended synergetics in Section 2, in Section 3.1 we will present a detailed discussion of the application of the extended synergetics model to child playing. In particular, we will present numerical simulations that were missing in the earlier work.

Subsequently, in Section 3.2, in analogy to the modeling strategy of the flow of actions during child play, we will discuss the flow of infant actions during infant-mother face-to-face communication. In this context, we will apply the model to experimental data that have been reported in the literature [17]. In other words, the focus in Section 3.2 is to show how the model can be related to experimental data reported in the literature.

\section{Modeling}

\section{1. $\quad$ Lotka-Volterra-Haken model}

We consider an infant or a child who can perform a repertoire of $N$ mutually exclusive actions. These actions, motor activities, or behaviors are interpreted as patterns. We do not model the patterns themselves (although this has been done e.g., in the context of pattern recognition and perception [18]). Rather, the objective is to model the evolution of the pattern amplitudes. Let $M_{1}, \ldots, M_{N}$ denote the amplitudes of the $N$ patterns under consideration. The pattern amplitudes $M_{k}$ are assumed to evolve in time $t$ such that $M_{1}(t), \ldots, M_{N}(t)$ represents a set of time-dependent amplitudes. The tuple $M_{1}(t), \ldots, M_{N}(t)$ represents a graph in a $N$-dimensional space. The graph describes the built-up of a particular action over time. In the transient period, typically two or more than two amplitudes $M_{k}$ are different from zero. In this transient period patterns compete with each other for being activated. The child or infant under consideration is undecided. Eventually, only one amplitude will survive the competition process and exhibit a finite value, whereas all other amplitudes will decay and become zero. Such a model is called a winner-takes-all model. The evolution equations for the $N$ amplitudes $M_{k}$ are given by [5]

$\frac{\mathrm{d}}{\mathrm{d} t} M_{k}=M_{k}\left(\lambda_{k}-M_{k}-g \sum_{j \neq k} M_{j}\right)$.

In Eq. (2) the parameters $\lambda_{k}$ denote growth parameters that describe the exponential increase (assuming $\lambda_{k}>0$ ) of the amplitudes when all amplitudes are close to zero. The parameter $g>1$ describes the strength of the competitive 
interactions between the patterns. Note that Eq. (2) is a special case of the Lotka-Volterra model for competitive species. Interestingly, by means of the variable transformation $\xi_{k}^{2}=M_{k}$ for $M_{k}>0$, see Ref. [15], the Lotka-Volterra model for $M_{k}$ can be transformed into the amplitude equations of the synergetic computer $[18,19]$ for $\xi_{k}$. Therefore, Eq. (2) is a synergetic model and will be referred to as Lotka-Volterra-Haken model. The Lotka-Volterra-Haken model unifies quantitative modeling approaches in psychology based on amplitude equations [18, 20] and population dynamics $[5,21,22]$.

In the stationary state, all amplitudes $M_{k}$ are constant (i.e., independent of time). As mentioned above, we are dealing with a winner-takes-all model. There will be only one amplitude $M_{k}$ different from zero; all others will be equal to zero. That is, Eq. (2) exhibits fixed points like

$M_{k}=\lambda_{k} \wedge M_{j \neq k}=0$,

assuming that $\lambda_{k}$ are constants and do not change with time. We refer to fixed points that satisfy Eq. (3) as "winner-takes-all" fixed points.

For the following discussion it is useful to consider relative amplitudes $M_{k, r e l}$, which correspond to amplitudes $M_{k}$ that are rescaled with respect to their stationary values $\lambda_{k}$ like

$M_{k, r e l}(t)=\frac{M_{k}(t)}{\lambda_{k}}$.

Having defined $M_{k, r e l}$, we introduce next a binary auxiliary variable $\left[M_{k}\right]$. In the first place, as we will see below, the auxiliary variable will make the model equations easier to read. The binary variable codes two mutually exclusive classes: a pattern can be present or absent. We refer to an amplitude as being "on" or "off" if the corresponding pattern is present or absent. From an implementation point of view, we use the integer values " 0 " and " 1 " for "on" and "off". The squared bracket of $M_{K}$ (i.e., $\left[M_{k}\right]$ ) denotes an operator that maps the real-valued amplitude $M_{k} \in \mathbb{R}$ to the binary auxiliary variable $\left[M_{k}\right] \in\{0,1\}$. The operator $[\cdot]$ is defined by

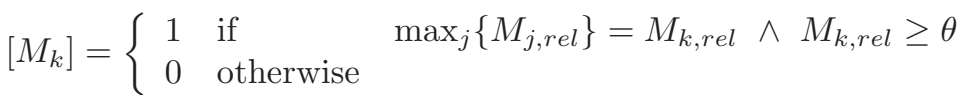

Here, $\theta$ is a relative threshold between 0 and 100 percent. The binary variable has a psychophysical interpretation. In perception, if $M_{k}$ denotes a particular percept out of $N$ possible percepts, then the pattern $k$ is perceived or experienced at the moment at which $M_{k}$ becomes "on". With respect to action, an infant or a child may start to execute a particular behavior $k$ when $M_{k}$ becomes "on" but not earlier.

\section{2. $\quad$ Extended synergetics}

According to the extended synergetic approach the parameters $\lambda_{k}$ are self-regulated parameters in the sense of definition (1). Explicitly, it is assumed that the parameters $\lambda_{k}$ satisfy evolution equations of the form [5]

$\frac{\mathrm{d}}{\mathrm{d} t} \lambda_{k}=-\frac{1}{\tau_{k}\left(\left[M_{k}\right]\right)}\left(\lambda_{k}-b_{k}\left(\left[M_{k}\right]\right)\right)+s \lambda_{k} \Gamma_{k}(t)$.

The variables $\tau_{k}(\cdot)>0$ are time constants. The smaller the value of a time constant for a given mode $k$, the faster does the growth parameter $\lambda_{k}$ evolve in time. The parameters $b_{k}$ are the stationary levels of the growth rates $\lambda_{k}$ in the absence of noise. That is, in the deterministic case, $s=0$, the parameters $\lambda_{k}$ converge to $b_{k}$ provided $b_{k}$ does not vary over time. The most right standing term in Eq. (6) is a multiplicative noise term. The parameter $s \geq 0$ corresponds to the noise strength $s^{2}$ of the noise source. The effective noise strength is modulated by the parameter $\lambda_{k}$. For small parameters $\lambda_{k}$ the parameter dynamics is only affected by a weak noise term, while for large parameters the noisy perturbations are large in magnitude. The function $\Gamma_{k}$ corresponds to a Langevin force $[23,24]$ normalized with respect to the Dirac delta function.

As indicated in Eq. (6), the time constants for parameter dynamics depend on the modes $M_{k}$ and my differ for the habituation and forgetting phases. We have

$\tau_{k}\left(\left[M_{k}\right]\right)=\left\{\begin{array}{lll}\tau_{k, \text { habituation }} & \text { if } & {\left[M_{k}\right]=1} \\ \tau_{k, \text { forgetting }} & \text { if } & {\left[M_{k}\right]=0}\end{array}\right.$.

Likewise, the fixed point values $b_{k}$ of the growth parameter dynamics depends on the pattern amplitudes. We put [5]

$b_{k}\left(\left[M_{k}\right]\right)=\left\{\begin{array}{lll}b_{0}-h & \text { if } & {\left[M_{k}\right]=1} \\ b_{0} & \text { if } & {\left[M_{k}\right]=0}\end{array}\right.$ 
with $b_{0}>0$. The parameter $h \geq 0$ measures the degree of self-inhibition. For $h=0$ there is no self-inhibition. Note that the auxiliary variable $\left[M_{k}\right]$ can be eliminated by using the definition (5). In doing so, a closed dynamical model for the amplitudes $M_{k}$ and the system parameters $\lambda_{k}$ can be derived that does not require to consider $\left[M_{k}\right]$ explicitly.

As shown in an earlier work [5] a winner-takes-all fixed point defined by Eq. (3) is asymptotically stable as long as $\lambda_{k}>\lambda_{j} / g$ for all $j \neq k$. However, if there exists at least one pattern $j$ with $\lambda_{k}<\lambda_{j} / g$ for all $j \neq k$, then the fixed point becomes unstable. Consequently, the critical condition at which a transition from a perceptualcognitive-behavioral pattern $k$ to another pattern $k^{\prime} \neq k$ occurs is given by

$k \rightarrow k^{\prime} \neq k$ if $\exists j: \lambda_{k}=\frac{\lambda_{j}}{g}$

Note that the dynamical system does not necessarily bifurcate from $k$ to $j$. The dynamical system can bifurcate to any pattern $k^{\prime}$ that is asymptotically stable and, consequently, satisfies $\lambda_{k^{\prime}}>\lambda_{j} / g$ for all $j \neq k^{\prime}$.

\subsection{Selection principle}

There is a selection principle that determines the bifurcations [5]. If $k$ becomes unstable then we have $\lambda_{k}<\lambda_{j} / g$ at least for one $j$ which implies that $\lambda_{k}<\lambda_{\max } / g$ with $\lambda_{\max }=\max _{w} \lambda_{w}$. The pattern that becomes unstable drops out of the so-called "stability band" [5, 14]. As mentioned above, the pattern $k^{\prime}$ that emerges satisfies $\forall j: \lambda_{k^{\prime}}>\lambda_{j} / g$, which implies $\lambda_{k^{\prime}}>\lambda_{\max } / g$. We say the pattern exhibits a growth parameter that is located in the "stability band". Consequently, at the transition point we have $\lambda_{k^{\prime}}>\lambda_{k}$. A transition occurs such that the growth rate of the pattern of the system is increased. In other words, in transitions between perceptual-cognitive-behavioral patterns we see that patterns are selected such that they come with a faster exponential growth rate. This selection principle does not only apply for the model (2) described above but also holds in general for Haken's amplitude equations of pattern formation systems [3, 14, 15] and other systems [25].

\subsection{4th Law by Swenson and Turvey}

The 4th law introduced by Swenson and Turvey states that transitions in perception-action systems and in intelligent systems in general occur in such a way that the rate of entropy production is maximized [26, 27]. That is, the 4th law defines a selection principle as well. Interestingly, for a large class of systems it has been shown that the attention parameters can be regarded as measures for the rate of entropy production [28, 29]. Consequently, for such systems the two selection principles, namely, the selection principle of faster growth rates and the selection principle of maximizing the rate of entropy production, have a common basis. If the growth rate parameters reflect the rate of entropy production, then the temporal ordering of action or the flow of action happens in such a way to maximize the rate of entropy production.

\subsection{Time scale separation and necessary condition for bifurcations}

If there is no self-inhibition $(h=0)$ then the parameters $\lambda_{k}$ are constants and transitions will not take place. In general, in order to arrive at a model that can produce transitions between patterns the magnitude of selfinhibition $h$ must be sufficiently large relative to the baseline level $b_{0}$ of the growth parameters. In order to make some semi-analytical predictions, we assume next that a time scale separation holds for amplitude and parameter dynamics in the sense that the amplitude dynamics evolves fast relative to the parameter dynamics: $\forall w=1, \ldots, N: \tau_{w} \gg 1 / \lambda_{w}$. Then we have $\lambda_{k}=b_{0}-h$ for the pattern $k$ that is "on" and $\lambda_{j}=b_{0}$ for the patterns $j$ that are "off". In this case, from the requirement $\exists j: \lambda_{k}<\lambda_{j} / g$ that a transition happens it follows that

$b_{0}-h<b_{0} / g$.

Solving for $h$ as a function of $b_{0}$ and $g$, we obtain

$h>b_{0} \frac{g-1}{g}$. 


\section{Results}

\subsection{Child playing}

Let us consider the leisure activities of a child at home. Let us assume the child may color a picture, may read a book, or watch television. That is, we distinguish between $N=3$ activities (behavioral modes). More explicitly, we consider the extended synergetic model defined by Eqs. (2)-(8) for $g>1$ and $N=3$ and interpret the fixed points $M_{1, s t}>0 \wedge M_{2, s t}=M_{3, s t}=0, M_{2, s t}>0 \wedge M_{1, s t}=M_{3, s t}=0$, and $M_{3, s t}>0 \wedge M_{1, s t}=M_{2, s t}=0$ in the 3D amplitude space as fixed points reflecting coloring, reading, and television watching, respectively.

Table 1: Simulation parameters

\begin{tabular}{lcccc}
\hline & $\mathrm{A}$ & $\mathrm{B}$ & $\mathrm{C}$ & $\mathrm{D}$ \\
\hline$\tau_{1}$ (on) [a.u.] & 10 & 10 & 10 & 10 \\
$\tau_{2}$ (on) [a.u.] & 10 & 10 & 10 & 10 \\
$\tau_{3}$ (on) [a.u.] & 10 & 30 & 10 & 30 \\
$s$ [a.u.] & 0.0005 & 0.05 & 0.0005 & 0.05 \\
$\tau_{1,2,3(\text { off })[\text { a.u. }]}$ & 10 & 10 & 10 & 10 \\
$g$ [a.u] & 1.5 & 1.5 & 1.5 & 1.5 \\
$b_{0}$ [a.u] & 6.0 & 6.0 & 6.0 & 6.0 \\
$h$ [a.u] & 3.0 & 3.0 & 3.0 & 3.0 \\
$\theta$ & 0.8 & 0.8 & 0.8 & 0.8 \\
\hline
\end{tabular}
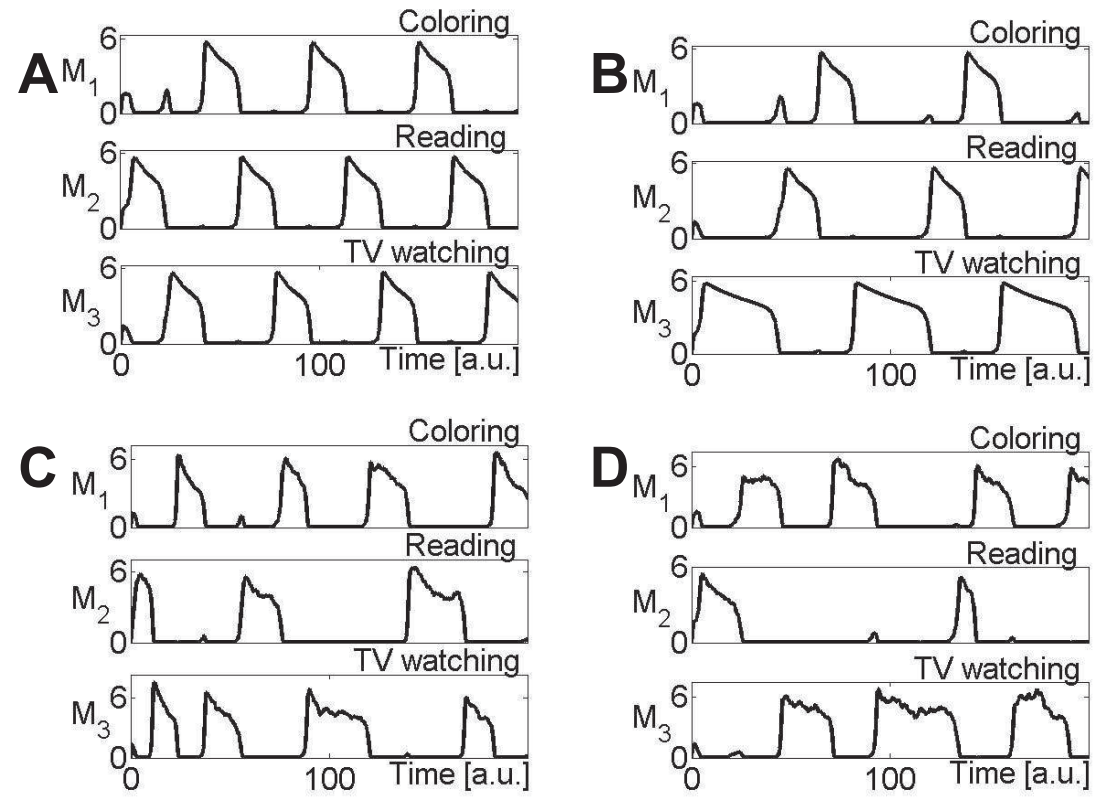

Figure 1: Amplitudes $M_{k}$ for $k=1,2,3$ as functions of time $t$ representing numerical solutions of the model given by Eqs. (2)-(8) under four different conditions A,B,C, and D. Amplitudes and time are measured in arbitrary units. In all simulations reported here and below an Euler-forward scheme with single time step of 0.001 was used. See text for details.

We conducted four computer experiments (labeled A,B,C, and D) in which we studied the impact of two independent variables. The first independent variable was the homogeneity of the time scale parameters $\tau_{k}$ for habituation. We considered the homogeneous case and the inhomogeneous case. We predicted that in the homogeneous case 
all activities have the same duration, whereas in the inhomogeneous case they have not. The second independent variable was the noise strength $s^{2}$. We consider a low and high noise case. We predicted that in the low noise case the temporal ordering of actions (flow of action) is periodic. In contrast, in the high noise case, the actions are selected in a random order.

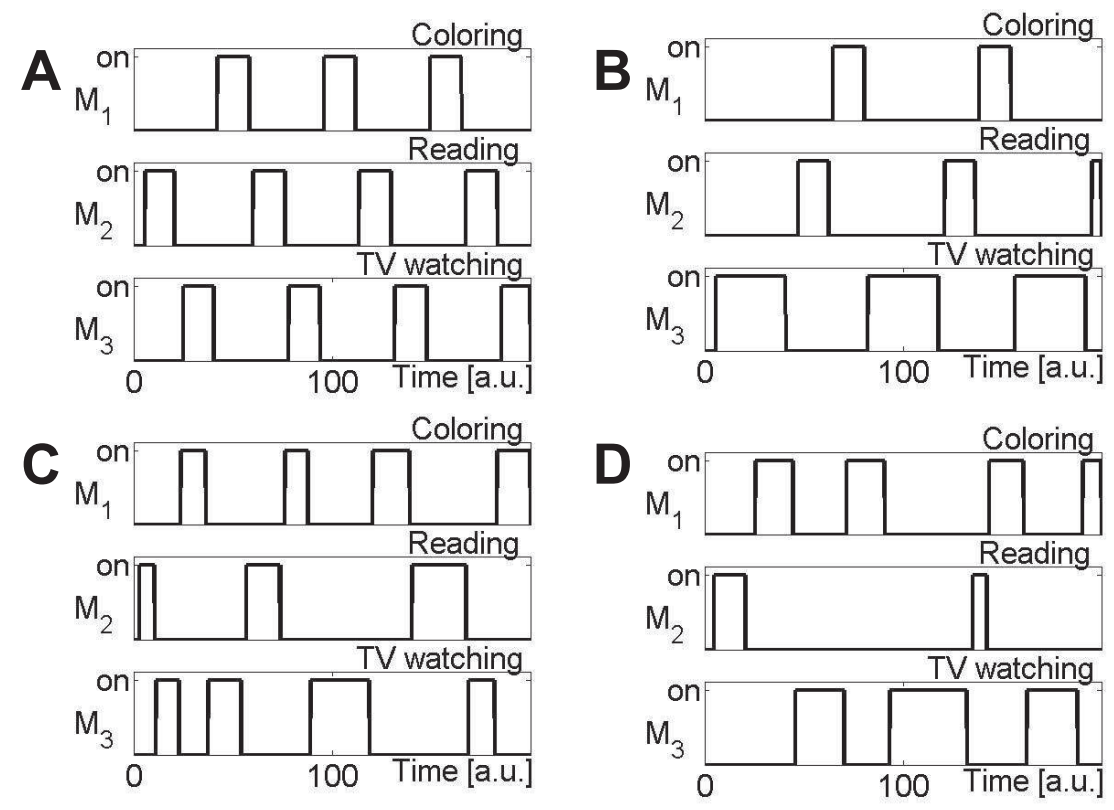

Figure 2: Auxiliary variables $\left[M_{k}\right]$ indicating the performance of a behavioral mode $k=1,2,3$ as functions of time t. $\left[M_{k}\right]$ were calculated from the trajectories $M_{k}(t)$ shown in Fig. 1 using Eq. (5).

The simulation parameters used in the four computer experiments are listed in Table 1. The simulation results for the four computer experiments A,B,C, and D are presented in Figs. 1-3. Figure 1 presents the amplitude dynamics and shows the amplitudes $M_{k}$ as functions of time. Figure 2 shows $\left[M_{k}\right]$ over time. Figure 3 shows the the parameter dynamics, that is, the growth rates $\lambda_{k}$ are presented as functions of time.

Let us discuss the first condition (A) in some detail. In the first condition, the simulation was conducted for homogeneous time scales and low noise (panels labeled A in Figs. 1-3).

Initially, the three activities competed with each other. The reading activity turned out to win the competition process. In the model, the amplitudes converged to the fixed point $M_{k}=\lambda_{k} \wedge M_{j \neq k}=0$ in the 3D amplitude space with $k=2$. Subsequently, the growth parameter $\lambda_{k}$ of the performed mode decayed monotonically. Eventually, the slowly evolving growth rate $\lambda_{k}$ of the performed mode (here $k=2$ ) dropped below the threshold $\lambda_{j} / g=b_{0} / g$ with $j \neq k$. At that moment in time, the slowly drifting location $M_{k}=\lambda_{k} \wedge M_{j \neq k}=0$ with $k=2$ became an unstable fixed point in the 3D amplitude space. The dynamics defined by the model (2)-(8) exhibited in the 3D amplitude space a bifurcation from the location $M_{k}=\lambda_{k} \wedge M_{j \neq k}=0$ to a winner-takes-all fixed point $M_{j}=\lambda_{j} \wedge M_{r \neq j}=0$ with $j \neq k$. In the simulations reported in Figs. 1-3 the bifurcation took place from $k=2$ to $j=3$, that is, the computer-simulated child began to watch TV. At that point in time, the habituation process of the performed activity (now TV-watching) started again. The activity became unstable and the computer-simulated child switched to another activity: reading. As predicted, the coloring-TV-watching-reading sequence repeated itself, see Figs. 1-3.

Our simulation demonstrates that under low noise, that is, when the dynamics is almost deterministic, the sequence of activities is periodic. As indicated above this can be predicted to some extend by means of theoretical reasonings. A deterministic dynamics can only show a small number of characteristic temporal patterns. The dynamics may converge to a fixed point. The dynamics may go to infinity in the long time limit. The dynamics may converge to a limit cycle behavior. The dynamics may settle down on a chaotic attractor. In our context, we can rule out the fixed point behavior and the blow-up behavior (system dynamics goes beyond any boundary). Consequently, the dynamical model (2)-(8) either exhibits a limit cycle behavior or a chaotic behavior. The numerical simulation 

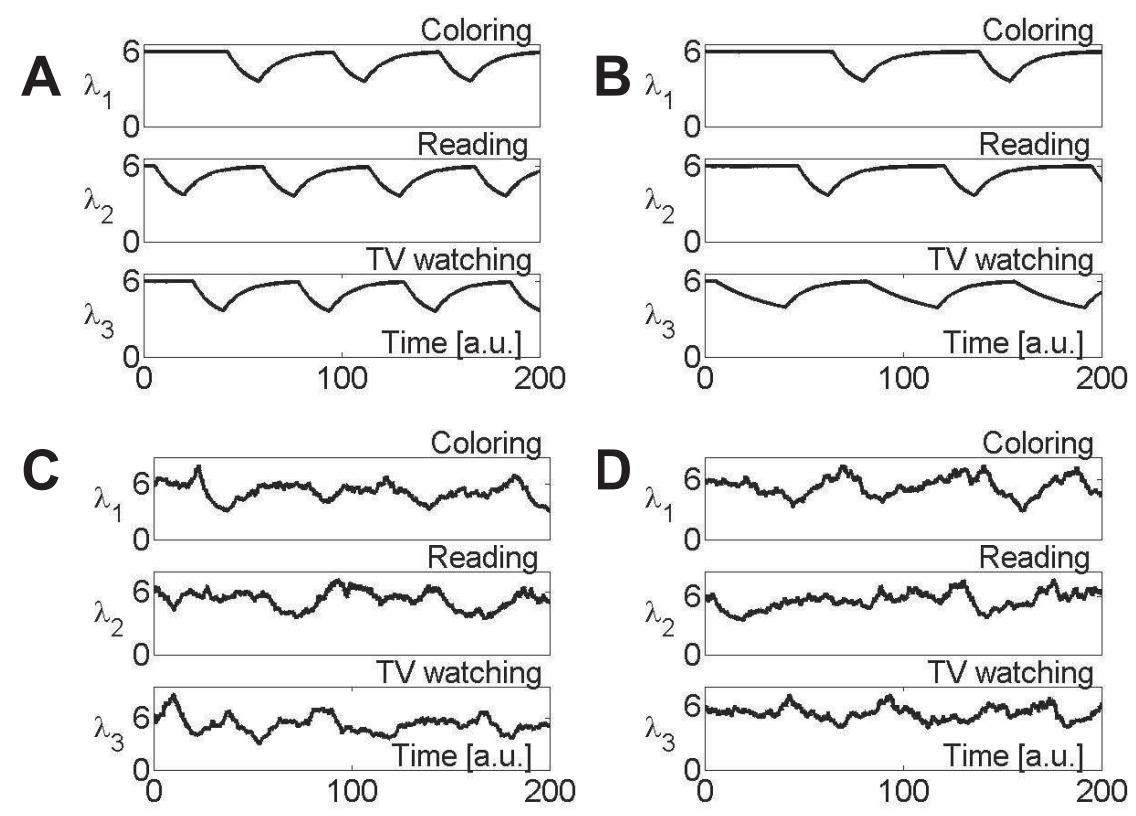

Figure 3: Growth rate parameters $\lambda_{k}$ associated with the behavioral modes $k=1,2,3$ as functions of time $t$ obtained by solving the model given by Eqs. (2)-(8) numerically under four different conditions A,B,C, and D. See text for details.

shows that at least for the parameters used in the computer experiment the dynamics is not chaotic. If the dynamics does not converge to a chaotic attractor, then we are left with the conclusion that the dynamics will exhibit after some transient period a limit cycle behavior.

The fact that the "three-activities-cycle" as a whole repeats itself is primarily of academic interest. That is, the observation helps us to understand better the properties of the model defined by Eqs. (2)-(8). In the real world, a child probably would not repeat the activities again. That is, only the first "three-activities-cycle" might be of interest when applying the model to real data.

Since $\lambda_{k}$ evolves slowly in time, the location $M_{k}=\lambda_{k} \wedge M_{j \neq k}=0$ is not a fixed point in the space $\mathbb{R}^{6}$ spanned by the vectors $\mathbf{M}=\left(M_{1}, M_{2}, M_{3}\right)$ and $\lambda=\left(\lambda_{1}, \lambda_{2}, \lambda_{3}\right)$. Only if the dynamics of the growth parameters $\lambda_{k}$ is ignored, then the location $M_{k}=\lambda_{k} \wedge M_{j \neq k}=0$ is a fixed point. Therefore, we refer to the location $M_{k}=\lambda_{k} \wedge M_{j \neq k}=0$ as fixed point in the 3D amplitude space. To repeat, from a mathematical point of view, the location $M_{k}=\lambda_{k} \wedge M_{j \neq k}=0$ is not a fixed point of the model defined by Eqs. (2)-(8). From a mathematical point of view the location $M_{k}=\lambda_{k} \wedge M_{j \neq k}=0$ is the intersection point of the nullclines for $\mathbf{M}=\left(M_{1}, M_{2}, M_{3}\right)$ in the $3 \mathrm{D}$ amplitude subspace of the state space $\mathbb{R}^{6}$ spanned by the vectors $\mathbf{M}=\left(M_{1}, M_{2}, M_{3}\right)$ and $\lambda=\left(\lambda_{1}, \lambda_{2}, \lambda_{3}\right)$.

Let us consider briefly the other three conditions B,C, and D. In the second condition (B), the noise was low just as in the first condition. However, we assumed that the habituation time scales were not all equal. In particular, we assumed that the habituation dynamics for TV watching would be slower than for the two other activities. Consequently, we put $\tau_{3}\left(\left[M_{3}\right]=\right.$ "on" $)>\tau_{1}\left(\left[M_{1}\right]=\right.$ "on" $)=\tau_{2}\left(\left[M_{1}\right]=\right.$ "on" $)$. As expected, we found that the activity with the longer habituation time scale (TV watching) was performed for a longer duration than the two alternative activities (coloring and reading). Mathematically speaking, since $\tau_{3}\left(\left[M_{3}\right]=\right.$ "on") was relatively large, the growth rate parameter $\lambda_{3}$ decayed relatively slow as compared to $\lambda_{1}$ and $\lambda_{2}$, see third rows of panels A and $\mathrm{B}$ in Fig. 3. Therefore, the time to reach the critical value $\lambda_{k}=\lambda_{\max } / g$ at which the performed activity became unstable was larger in the case of the TV watching (mode $k=3$ ) than in the case for the other behaviors (modes $k=1,2)$.

In the third condition $(\mathrm{C})$, we considered homogeneous habituation time scales just as in the first condition (A) but the noise strength $s^{2}$ was relatively large. As expected, the repetitive pattern of the flow of actions that was observed under the low noise condition (A) was broken. The sequence of actions showed a more or less random order as can be seen by comparing the first and second rows of panels A and C in Figs. 1 and 2. 
Finally, in the fourth condition (D), we studied child play involving a non-homogeneous set of habituation time scales and strong, noisy perturbations. According to the extended synergetic model defined by Eqs. (2)-(8) we found that the two novel aspects observed under conditions (B) and (C) were both present under condition (D). That is, the activity with the longer habituation time scale was performed for a longer duration and the flow of actions showed a random order rather than a periodic one, see first and second rows of panels A and D in Figs. 1 and 2 .

\subsection{Infant actions during infant-mother face-to-face communication}

Early infant-mother face-to-face communication covers the period of the first few months of an infant's life. The quality of the infant-mother communication during that period is important for the development of the infantmother relationship as a whole [30]. Moreover, during that period (typically at the end of the second month) infants undergo a "developmental transition". The transition is characterized by an increase of the repertoire of actions that are available to the infant and are performed by the infant [31]. In particular, after the transition the infant has acquired the ability to smile in social contexts [32]. The transition is a developmental milestone at which a new type of dialog between the infant and the mother becomes possible [33]. More explicitly, a recent study by Lavelli and Fogel [17] has focused on six elementary infant behaviors that can be observed during infantmother face-to-face communication. For sake of simplicity, these six behaviors will be grouped in what follows into three categories: the infant has the gaze elsewhere (i.e., does not face the mother), the infant shows attention to the mother, the infant smiles to the mother. From the data reported in the study by Lavelli and Fogel [17], the durations of the three type of actions as performed during 3 minute periods of face-to-face communication can be calculated. For infants at the age of 1,8 , and 12 weeks the durations are shown in Table 2.

Table 2: Durations of infant actions as functions of age as obtained by a re-analysis of the data reported by Lavelli and Fogel [17]

\begin{tabular}{cccc}
\hline Age & Gaze elsewhere [sec] & Attention [sec] & Smile [sec] \\
\hline 1 & 160.0 & 20.0 & 0 \\
8 & 60.5 & 67.0 & 52.5 \\
12 & 74.0 & 45.5 & 60.5 \\
\hline
\end{tabular}

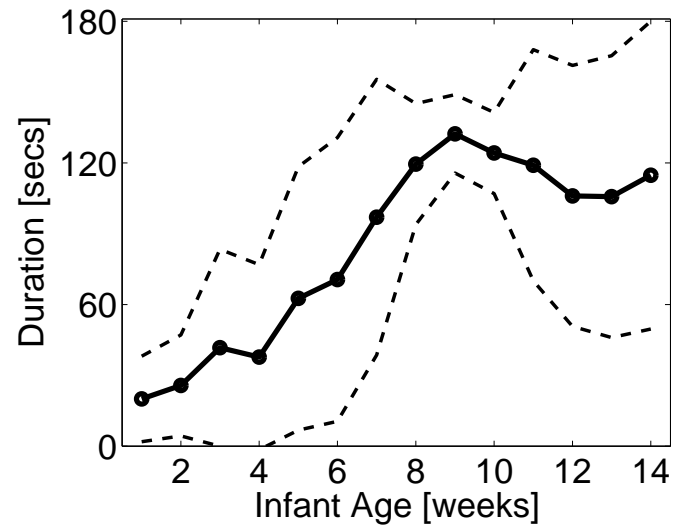

Figure 4: Duration of infant-mother face-to-face communication observed during 3 minutes recording periods as a functions of age.

If the gaze of the infant is not focused on the mother, we consider this as a sign that the infant is not engaged in any form of face-to-face communication. Consequently, if we sum up the times during which an infant either shows attention or is smiling to the mother, then we obtain a measure for the duration of the face-to-face communication of the infant during an observed three minutes period. Fig. 4 illustrates the face-to-face communication duration thus obtained as a function of age. The transition at the end of the second month (i.e., at about 8 weeks) is clearly visible. 
Table 3: Simulation parameters as function of age. Other parameters independent of age: $\tau_{k}($ "off" $)=100$ sec, $g=1.5, b_{0}=6, h=3, \theta=0.8, s=0.05$.

\begin{tabular}{cccc}
\hline Age & $\tau_{1}$ ("on") [sec] & $\tau_{2}$ ("on") [sec] & $\tau_{3}$ ("on") $[\mathrm{sec}]$ \\
\hline 1 & $(160+85) / 180^{*} 100$ & $(20-15) / 180^{*} 100$ & 100 \\
8 & $60.5 / 180^{*} 100$ & $67.0 / 180^{*} 100$ & $52.5 / 180^{*} 100$ \\
12 & $74.0 / 180^{*} 100$ & $45.5 / 180^{*} 100$ & $60.5 / 180^{*} 100$ \\
\hline
\end{tabular}

Our objective is to model the infant behavior in infant-mother face-to-face interactions for infants before and after the transition. We distinguish between the three aforementioned actions. We consider the extended synergetic model defined by Eqs. (2)-(8) for $g>1$ and $N=3$ and interpret the fixed points $M_{1, s t}>0 \wedge M_{2, s t}=M_{3, s t}=0$, $M_{2, s t}>0 \wedge M_{1, s t}=M_{3, s t}=0$, and $M_{3, s t}>0 \wedge M_{1, s t}=M_{2, s t}=0$ (i.e., the intersection points of the amplitude nullclines) in the 3D amplitude (sub)space as locations reflecting "gaze elsewhere", "attention", and "smile".
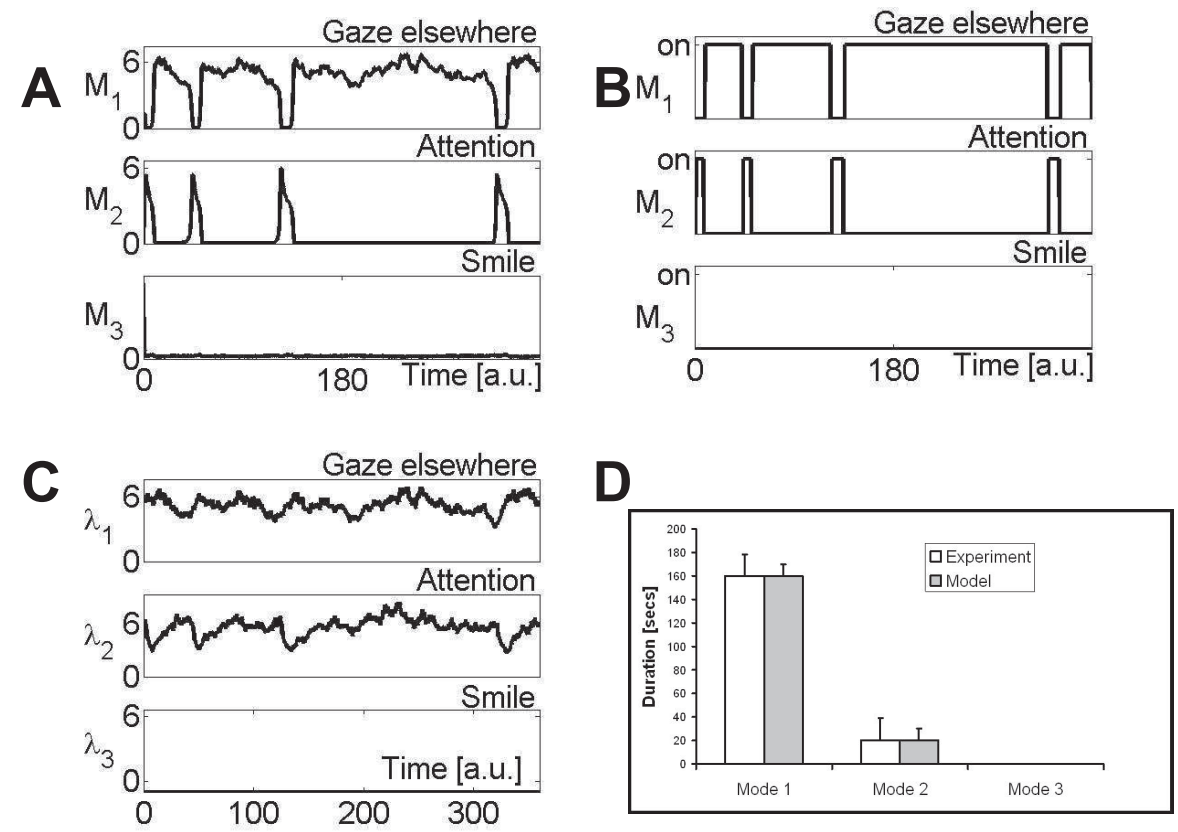

Figure 5: Simulation results for 1 week old infants. Panels A,B,C: simulation of an individual infant. Panel D: comparison of experimental data with model predictions obtained from a sample of 10 computer-simulated infants. Error bars correspond to standard deviations. See text for details.

We conducted three computer experiments in which we matched the model parameters to the observed durations (see Table 2) of the three activities for infants at the age of 1, 8, and 12 weeks. Since smiling was not observed in 1 week old infants, for the first computer experiment we put $\lambda_{3}=-1$. As a result, the amplitude $M_{3}$ converged to zero in any case. Other than that for all three experiments we kept all parameters constant expect for the timing parameters $\tau_{k}$ ("on"). The simulation parameters used in the three computer experiments are listed in Table 3. The noise level was chosen to be relatively high. The simulation period was two times the period used in the experimental study by Lavelli and Fogel [17]. The simulation results are presented in Figs. 5-7. In all figures, panels $\mathrm{A}, \mathrm{B}$, and $\mathrm{C}$ show the amplitudes $M_{k}$, the auxiliary variables $\left[M_{k}\right]$, and the growth parameters $\lambda_{k}$ as functions of time in a 360 seconds period. For each age group a total of 10 infants were simulated. From the trajectories an appropriate rescaled measure for the duration in a 180 seconds period was derived for each infant. Subsequently, 

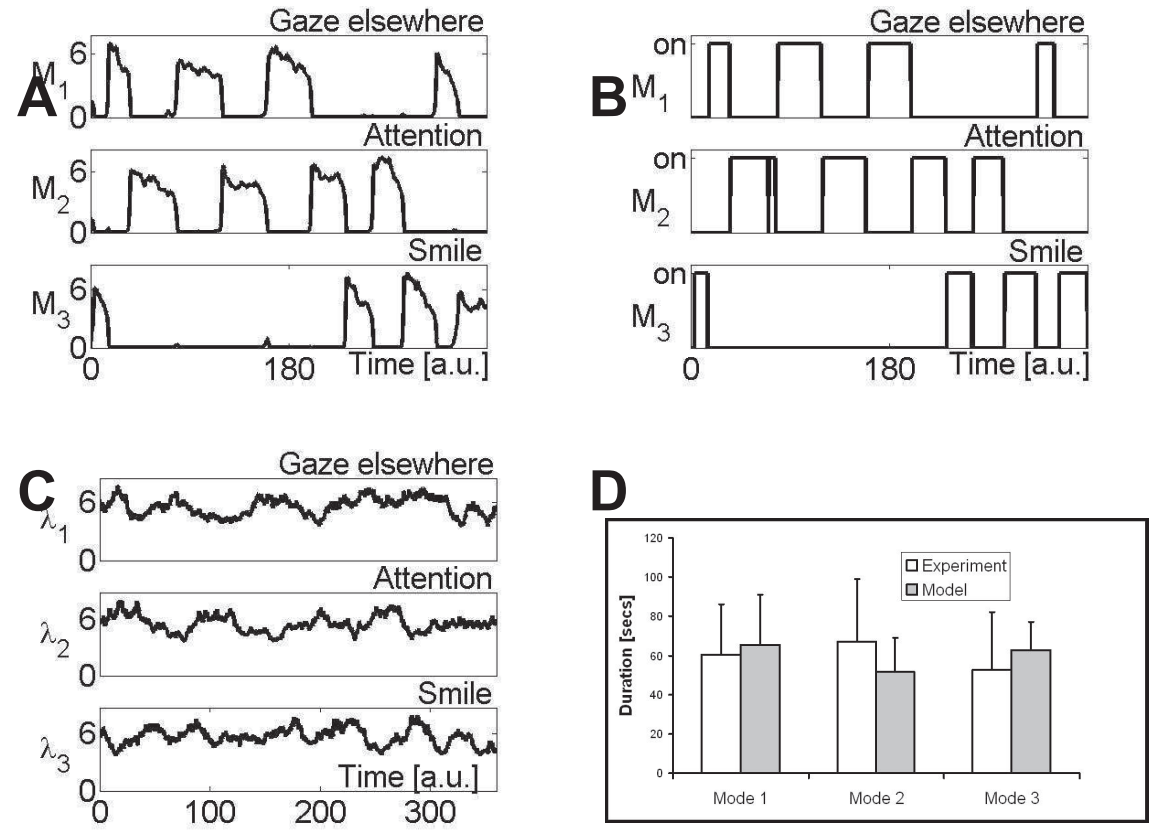

Figure 6: As in Fig. 5 but for infants at the age of 8 weeks.

sample mean values across the 10 infants were calculated. Panels D in Figs. 5-7 show for $k=1,2,3$ the durations as predicted by the extended synergetic model given by Eqs. (2)-(8) and the durations observed in the experiments, see Table 2 . We see that the experimental data can be fitted well by only manipulating the relaxation times $\tau_{k}($ "on") of the habituation dynamics.

\section{Conclusion}

We presented a general model for child play, on the one hand, and infant behavior during infant-mother face-to-face communication, on the other. The model was based on extended synergetics. That is, a key ingredient was the coupling between amplitude dynamics and system parameter dynamics. As system parameters we used the growth rate parameters of the amplitudes. We argued that the growth rates dynamics reflects habituation of a performed activity. The habituation process makes the performed activity unstable. As a result, a bifurcation towards a new, stable activity can be observed. Note that this bifurcation is due to a self-regulation of a system parameter as defined by definition (1).

Table 4: Examples of models based on extended synergetics using either self-inhibition or cross-excitation or both.

\begin{tabular}{llll}
\hline Flow of action & Self-inhibition & Cross-excitation & Ref. \\
\hline Scene decomposition & $\mathrm{x}$ & - & {$[6]$} \\
Oscillatory perception of ambivalent figures & $\mathrm{x}$ & - & {$[8,9,34]$} \\
Verbal transformation effect & $\mathrm{x}$ & - & {$[10,11]$} \\
Negative hysteresis in grasping transitions & $\mathrm{x}$ & - & {$[13]$} \\
OCD rituals & $\mathrm{x}$ & $\mathrm{x}$ & {$[5,35]$} \\
Child playing & $\mathrm{x}$ & - & Present study and Ref. [5] \\
Infant behavior (simplified) & $\mathrm{x}$ & - & Present study \\
\hline
\end{tabular}




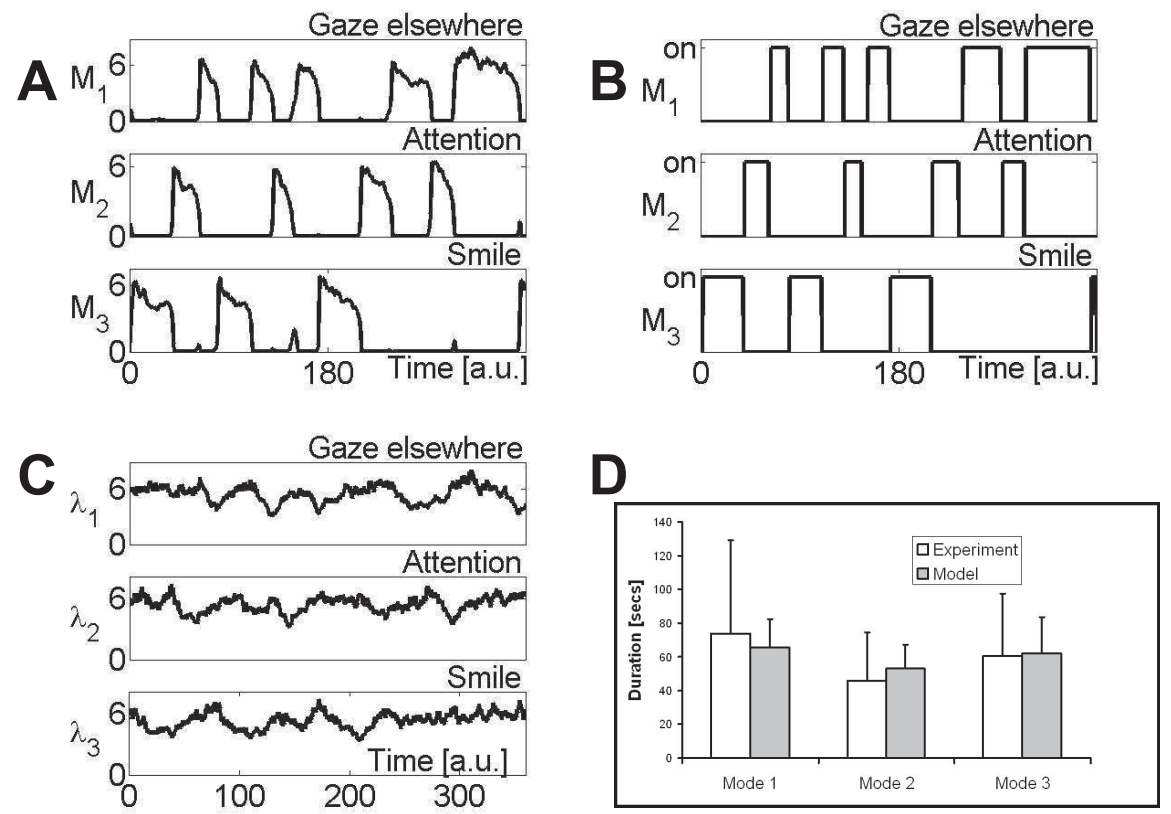

Figure 7: As in Fig. 5 but for infants at the age of 12 weeks.

The model also involves some kind of forgetting. When the behavior $j$ is no longer performed, then the growth rate parameter of the amplitude of the behavior $j$ relaxes back to a default level. In this sense, the infant starts to forget that he or she had performed the activity $j$.

Nonlinear physics models based on extended synergetics that involve self-regulated system parameters and capture self-inhibition processes have been discussed in several studies, see Table 4 . As mentioned above, in these examples, it is assumed that habituation or inhibition makes the performed activity (or the experienced percept) unstable. However, from a theoretical perspective, cross-excitation can have the same effect. In fact, cross-excitation was used to model that rituals of obsessive-compulsive-disorder (OCD) patients are executed in a particular order $[5,35]$. Both mechanisms make the performed activity (or the experienced percept) unstable. Selfinhibition in the presence of noise does not specify which mode emerges at the bifurcation point. In contrast, crossexcitation favors the emergence of the excited mode and consequently specifies a bifurcation route or "direction".

In the context of modeling child play we investigated two independent variables: the homogeneity of the time constants of the habituation process and the strength of noise acting on the system parameter dynamics (growth rate parameters). We found two main effects. First, if the time constants are not homogeneous then the actions are performed with unequal durations. Second, if the noise strength is increased then the flow of actions becomes random in the sense that actions are performed in a random order rather than a periodic one. The question at issue is whether or not there is an interaction effect. This question may be examined in future studies. Here, we only want to mention that since the parameter dynamics involves a multiplicative noise force it might be the case that the strength of the noise source has an impact on the mean value of a growth parameter. In this case, it is likely that the noise that perturbs the dynamics of a growth parameter of an on-going activity also affects the duration of the activity.

In the case of the infant behavior during infant-mother face-to-face communication we found that the age-related shift towards activities that support communication (here: attention and smile) can be understood by a change in the spectrum of time constants of the habituation dynamics. In particular, the developmental transition at about 8 weeks can be captured by assuming that time constants change qualitatively on the relatively slow time scale of infant maturation such that the communication-supportive activities are performed for longer durations. 
The infant behavior model developed above is a simplified model in which the actions of the mother are ignored. Future studies may be devoted to construct more comprehensive models that can address not only the behavior of the infant but also the behavior of the mother. Such models would describe the behavior of infant-mother-dyads. In this case, it seems to be promising not only to consider within-person self-inhibition but also to take between-persons cross-excitation into account similar to the within-person cross-excitation mentioned above (see also Table 4).

\section{Acknowledgments}

Preparation of this manuscript was supported in part by National Science Foundation under the INSPIRE track, grant BCS-SBE-1344275.

\section{References}

[1] J. A. S. Kelso, Dynamic patterns - The self-organization of brain and behavior, MIT Press, Cambridge, 1995.

[2] H. Haken, Principles of brain functioning, Springer, Berlin, 1996.

[3] T. D. Frank, J. van der Kamp, G. J. P. Savelsbergh, On a multistable dynamic model of behavioral and perceptual infant development, Dev. Psychobiol. 52 (2010) 352-371.

[4] T. D. Frank, Motor development during infancy: a nonlinear physics approach to emergence, multistability, and simulation, in: A. M. Columbus (Ed.), Advances in psychology research, Vol. 83, Nova Publ., New York, 2011, pp. Chap. 9 (pp. 143-160).

[5] T. D. Frank, Secondary bifurcations in a Lotka-Volterra model for n competitors with applications to action selection and compulsive behaviors, Int. J. Bif. and Chaos (in press)

[6] A. Fuchs, H. Haken, Pattern recognition and associative memory as dynamical processes in a synergetic system. I. Translational invariance, selective attention and decomposition of scene, Biol. Cybern. 60 (1988) 17-22.

[7] A. Fuchs, H. Haken, Pattern recognition and associative memory as dynamical processes in a synergetic system. II. Decomposition of complex scenes, simultaneous invariance with respect to translation, rotation, and scaling, Biol. Cybern. 60 (1988) 107-109.

[8] T. Ditzinger, H. Haken, Oscillations in the perception of ambigious patterns: a model based on synergetics, Biol. Cybern. 61 (1989) 279-287.

[9] T. Ditzinger, H. Haken, Impact of fluctuations on the recognition of ambiguous patterns, Biol. Cybern. 63 (1990) 453-456.

[10] T. Ditzinger, B. Tuller, J. A. S. Kelso, Temporal patterning in an auditory illusion: the verbal transformation effect, Biol. Cybern. 77 (1997) 23-30.

[11] T. Ditzinger, B. Tuller, H. Haken, J. A. S. Kelso, A synergetic model for the verbal transformation effect, Biol. Cybern. 77 (1997) 31-40.

[12] B. Tuller, P. Case, M. Ding, J. A. S. Kelso, The nonlinear dynamics of speech recognition, J. Exp. Psychol. - Hum. Percept. Perform. 20 (1994) 3-16.

[13] S. M. Lopresti-Goodman, M. T. Turvey, T. D. Frank, Negative hysteresis in the behavioral dynamics of the affordance "graspable", Attention, Perception, and Psychophysics 75 (2013) 1075-1091.

[14] T. D. Frank, On a multistable competitive network model in the case of an inhomogeneous growth rate spectrum with an application to priming, Phys. Lett. A 373 (2009) 4127-4133.

[15] T. D. Frank, Psycho-thermodynamics of priming, recognition latencies, retrieval-induced forgetting, priming-induced recognition failures and psychopathological perception, in: N. Hsu, Z. Schütt (Eds.), Psychology of priming, Nova Publ., New York, 2012, pp. Chap. 9 (pp. 175-204).

[16] T. D. Frank, From systems biology to systems theory of bipolar disorder, in: F. Miranda (Ed.), Systems theory: perspectives, applications and developments, Nova Publ., New York, 2014, pp. Chap. 2 (pp. 17-36).

[17] M. Lavelli, A. Fogel, Interdyad differences in early mother-infant face-to-face communication: real-time dynamics and developmental pathways, Developmental Psychology 49 (2013) 2257-2271. 
[18] H. Haken, Synergetic computers and cognition, Springer, Berlin, 1991.

[19] T. D. Frank, New perspectives on pattern recognition algorithm based on Haken's synergetic computer network, in: M. D. Fournier (Ed.), Perspective on pattern recognition, Nova Publ., New York, 2011, pp. Chap. 7 (pp. 153-172).

[20] S. M. Lopresti-Goodman, M. T. Turvey, T. D. Frank, Behavioral dynamics of the affordance "graspable", Attention, Perception, and Psychophysics 73 (2011) 1948-1965.

[21] J. Kriz, Synergetics in clinical psychology, in: H. Haken, M. Stadler (Eds.), Synergetics in cognition, Springer, Berlin, 1990, pp. 393-404.

[22] M. I. Rabinovich, M. K. Muezzinoghu, I. Strigo, A. Bystritsky, Dynamic principles of emotion-cognition interaction: mathematical images of mental disorders, PLoS ONE 5 (2010) e12547.

[23] H. Risken, The Fokker-Planck equation — Methods of solution and applications, Springer, Berlin, 1989.

[24] T. D. Frank, Nonlinear Fokker-Planck equations: Fundamentals and applications, Springer, Berlin, 2005.

[25] T. D. Frank, Decision making in physical intelligent systems regulated by growth rate factors, Computer and Information Science 7 (2014) 55-64.

[26] R. Swenson, M. T. Turvey, Thermodynamic reasons for perception-action cycles, Ecol. Psychol. 3(4) (1991) 317-348.

[27] M. T. Turvey, C. Carello, On intelligence from first principles: guidelines for the inquiry into the hypothesis of physical intelligence, Ecological Psychology 24 (2012) 3-32.

[28] T. D. Frank, Rate of entropy production as a physical selection principle for mode-mode transitions in non-equilibrium systems: with an application to a non-algorithmic dynamic message buffer, European Journal of Scientific Research 54 (2011) 59-74.

[29] T. D. Frank, Pumping and entropy production in non-equilibrium drift-diffusion systems: a canonical-dissipative approach, European Journal of Scientific Research 46 (2010) 136-146.

[30] C. H. H, A. Fogel, Stability and transitions in mother-infant face-to-face communication during the first 6 months: a microhistorical approach, Developmental psychology 39 (2003) 1061-1082.

[31] N. Herschkowitz, J. Kagan, K. Zilles, Neurobiological bases of behavioral development in the first year, Neuropediatrics 28 (1997) 296-306.

[32] P. H. Wolff, Behavioral states and the expressions of emotion in early infancy, University of Chicago Press, Chicago, 1987.

[33] T. B. Brazelton, H. Als, Four early stages in the development of mother-infant interaction, Psychoanalytical Study of the Child 34 (1979) 349-369.

[34] T. D. Frank, Multistable perception in schizophrenia: a model-based analysis via coarse-grained order parameter dynamics and a comment on the 4th law, Universal Journal in Psychology 2 (2014) 231-240.

[35] T. D. Frank, Action flow in obsessive-compulsive disorder rituals: a model based on extended synergetics and a comment on the 4th law, Journal of Advances in Physics 5 (2014) 845-853. 